\title{
Probing the supersymmetric parameter space by weakly interacting massive particle direct detection
}

\author{
A. Bottino* \\ Dipartimento di Fisica Teorica, Università di Torino and INFN, Sez. di Torino, Via P. Giuria 1, I-10125 Torino, Italy \\ F. Donato ${ }^{\dagger}$ \\ Laboratoire de Physique Théorique LAPTH, B.P. 110, F-74941 Annecy-le-Vieux Cedex, France \\ and INFN, Sede di Presidenza, 00186 Roma, Italy \\ N. Fornengo $0^{\ddagger}$ and S. Scopel ${ }^{\S}$ \\ Dipartimento di Fisica Teorica, Università di Torino and INFN, Sez. di Torino, Via P. Giuria 1, I-10125 Torino, Italy \\ (Received 25 October 2000; published 1 May 2001)
}

\begin{abstract}
We discuss to what extent the present experiments of direct search for weakly interacting massive particles (WIMPs), when interpreted in terms of relic neutralinos, probe interesting regions of the supersymmetric parameter space, which are also being progressively explored at accelerators. Our analysis is performed in a number of different supersymmetric schemes. We derive the relevant neutralino cosmological properties, locally and on the average in the universe. We prove that part of the supersymmetric (SUSY) configurations probed by current WIMP experiments entails relic neutralinos of cosmological interest. The main astrophysical and particle physics uncertainties, relevant for a proper comparison between theory and experimental data, are stressed and taken into account.
\end{abstract}

DOI: 10.1103/PhysRevD.63.125003

PACS number(s): 11.30.Pb, 12.60.Jv, 14.80.Ly, 95.35.+d

\section{INTRODUCTION}

As first noticed in Ref. [1], in the last few years the experiments of direct search for weakly interacting massive particles (WIMP) [2] have already reached a sensitivity which allows the exploration of regions of the supersymmetric (SUSY) parameter space, which are also progressively investigated at accelerators. This property is manifest, when the experimental results are interpreted in terms of relic neutralinos [1].

The probing of the SUSY parameter space by WIMP direct searches is even more sizable at present, with the sensitivities of experiments [3,4]; a comparative discussion of the experimental features and implications of the DAMA [3] and CDMS [4] experiments may be found in Ref. [5]. Detailed studies of the possible interpretation of the annualmodulation effect [3] in terms of relic neutralinos have been reported in [6-9]. Comparisons of the experimental data of Ref. [3] with SUSY calculations have also been performed in Refs. [10-15].

In the present paper we intend to clarify the actual capability of WIMP direct searches by exploring in a systematic way different realizations of minimal supersymmetric models and showing their intrinsic differences in the prediction of neutralino rates and relic abundance. Specifically, we will consider two different implementations of a supergravity scheme with parameters defined at the grand unification scale and an effective supersymmetric model defined at the

\footnotetext{
*Email address: bottino@to.infn.it

†Email address: donato@lapp.in2p3.fr

*Email address: fornengo@to.infn.it

§ Email address: scopel@to.infn.it
}

electroweak scale. Our analyses are performed in the light of the following relevant points: (i) current uncertainties in astrophysical properties, (ii) uncertainties in hadronic quantities, (iii) new bounds from CERN $e^{+} e^{-}$collider LEP searches for Higgs and supersymmetric particles, (iv) updated determinations of cosmological parameters.

Let us start by recalling that the determination of the sensitivity range of an experiment of WIMP direct search in terms of the WIMP mass and of the WIMP-nucleon cross section rests on a number of crucial assumptions, since it depends both on the distribution function of the WIMPs in the halo and on the nature of the relic particle.

A WIMP direct experiment provides a measurement (or an upper bound) of the differential event rate

$$
\frac{d R}{d E_{R}}=N_{T} \frac{\rho_{W}}{m_{W}} \int d \vec{v} f(\vec{v}) v \frac{d \sigma}{d E_{R}}\left(v, E_{R}\right)
$$

where $N_{T}$ is the number of the target nuclei per unit of mass, $m_{W}$ is the WIMP mass, $\rho_{W}$ is the local WIMP matter density, $\vec{v}$ and $f(\vec{v})$ denote the WIMP velocity and velocity distribution function in the Earth frame $(v=|\vec{v}|)$ and $d \sigma / d E_{R}$ is the WIMP-nucleus differential cross section. The nuclear recoil energy is given by $E_{R}=m_{\text {red }}^{2} v^{2}\left(1-\cos \theta^{*}\right) / m_{N}$, where $\theta^{*}$ is the scattering angle in the WIMP-nucleus center-of-mass frame, $m_{N}$ is the nuclear mass, and $m_{\text {red }}$ is the WIMPnucleus reduced mass. Equation (1) refers to the case of a monoatomic detector, like the Ge detectors. Its generalization to more general situations, like for instance the case of $\mathrm{NaI}$, is straightforward. In what follows $\rho_{W}$ will be factorized in terms of the local value for the total nonbaryonic dark matter density $\rho_{l}$ and of the fractional amount of density, $\xi$, contributed by the candidate WIMP, i.e., $\rho_{W}=\xi \cdot \rho_{l}$. For $\rho_{l}$ we use the range $0.2 \mathrm{GeV} \mathrm{cm}^{-3} \leqslant \rho_{l} \leqslant 0.7 \mathrm{GeV} \mathrm{cm}^{-3}$, where the upper side of the range takes into account the 
possibility that the matter density distribution is not spherical, but is described by an oblate spheroidal distribution $[16,17]$.

The WIMP-nucleus differential cross section may conveniently be split into a coherent part and a spin-dependent one

$$
\frac{d \sigma}{d E_{R}}=\left(\frac{d \sigma}{d E_{R}}\right)_{C}+\left(\frac{d \sigma}{d E_{R}}\right)_{S D},
$$

whose generic features are discussed in the seminal paper of Ref. [18]. To compare theoretical expectations with experimental data, and experimental data of different detectors among themselves, it is useful to convert the WIMP-nucleus cross section into a WIMP-nucleon cross section. This procedure is feasible independently of the nuclear model and of the specific nature of the WIMP only under the hypothesis that the coherent cross section is dominant and the WIMP couples equally to protons and neutrons (at least approximately) [1]. Under this assumption, the WIMP-nucleus cross section may be expressed in terms of a WIMP-nucleon scalar cross section $\sigma_{\text {scalar }}^{\text {(nucleon) }}$ as

$$
\frac{d \sigma}{d E_{R}} \simeq\left(\frac{d \sigma}{d E_{R}}\right)_{C} \simeq \frac{F^{2}(q)}{E_{R}^{\max }}\left(\frac{1+m_{W} / m_{p}}{1+m_{W} / m_{N}}\right)^{2} A^{2} \sigma_{\text {scalar }}^{\text {(nucleon) }},
$$

where $m_{p}$ and $m_{N}$ are the proton and nucleus mass, $A$ is the nuclear mass number, $E_{R}^{\max }$ is the maximal recoil energy, and $F(q)$ is the nuclear form factor for coherent interactions. This form factor is usually parametrized in the Helm form [19]; however, precise evaluations of the event rates may require specific nuclear calculations for each target nucleus. In the rest of this paper we assume that the WIMP interaction with the nuclei of the detector is dominated by coherent effects, so that a WIMP-nucleon scalar cross section may be derived from the WIMP-nucleus cross section by use of Eq. (3).

Now, coming back to the general expression in Eq. (1), we stress that extracting an information about the WIMPnucleus cross sections from the experimental data requires the use of a specific expression for the velocity distribution function $f(\vec{v})$ [notice that in writing Eq. (3) we have already made the assumption that the WIMP phase-space distribution function may be factorized as $\rho \cdot f(\vec{v})$, and this is certainly not the most general case [16]]. The usual choice for $f(\vec{v})$ is the isotropic Maxwell-Boltzmann distribution in the galactic rest frame, as derived from the isothermal-sphere model.

However, recent investigations have shown that deviations from this standard scheme, either due to a bulk rotation of the dark halo [20,21] or to an asymmetry in the WIMP velocity distribution [22-24], influence the determination of the WIMP-nucleus cross sections from the experimental data in a sizable way. In Ref. [23] also triaxial matter distributions are considered; in the present paper deviation from sphericity in the WIMP matter distributions are taken into account only through the physical range allowed for the value of $\rho_{l}$ [see our previous comment on $\rho_{W}$ after Eq. (1)]. In a typical plot, where the WIMP-nucleus cross section is given in terms of the WIMP mass, the effect introduced by the mentioned deviations from the Maxwell-Boltzmann distribution is generically to elongate the contours towards larger values of $m_{W}$. This is for instance the case for the annual-modulation region of the DAMA Collaboration [3]. In Fig. 3 of Ref. [8] it is shown that, by implementing the dark halo with a bulk rotation according to the treatment in Ref. [21], the annual-modulation region moves towards larger values of the WIMP mass, with an elongation which brings the right-hand extreme from the value of $\sim 150 \mathrm{GeV}$ to $\sim 200 \mathrm{GeV}$. A similar effect is obtained by introducing an asymmetry in the WIMP velocity distribution $f(\vec{v})$, Fig. 4 of Ref. [24] illustrates this point. Notice that this asymmetry effect also pushes somewhat downwards the annualmodulation region. We emphasize that all these effects are extremely important when experimental results of WIMP direct detection are being compared with theoretical models for specific candidates. This point has been overlooked in most analyses in terms of relic neutralinos [25].

In the present paper we focus our analysis to the WIMP mass range which, in the light of the present experimental data $[3,4]$ and of the previous considerations on the astrophysical uncertainties, appears particularly appealing:

$$
40 \mathrm{GeV} \leqslant m_{W} \leqslant 200 \mathrm{GeV} \text {. }
$$

Let us notice that the mass range of Eq. (4) is quite appropriate for neutralinos. Actually, the lower extreme is indicative of the LEP lower bound on the neutralino mass $m_{\chi}$ (in the calculations performed in the present work the actual lower bound for $m_{\chi}$, dependent on the other SUSY parameters, is employed, according to the constraints given in [26]). As for the upper extreme, we notice that, though a generic range for $m_{\chi}$ might extend up to about $1 \mathrm{TeV}$, requirements of no excessive fine-tuning [27] would actually favor an upper bound of order $200 \mathrm{GeV}$, in accordance with Eq. (4).

In what follows we will discuss the discovery potential of WIMP direct searches for WIMPs in the mass range of Eq. (4). Particular attention will be paid to capabilities of the present experiments; their sensitivity range, in case of WIMPs whose coherent interactions with ordinary matter are dominant over the spin-dependent ones, may be stated, in terms of the quantity $\xi \sigma_{\text {scalar }}^{\text {(nucleon) }}$, as $[3,4]$

$$
4 \times 10^{-10} \text { nbarn } \leqslant \xi \sigma_{\text {scalar }}^{\text {(nucleon) }} \leqslant 2 \times 10^{-8} \text { nbarn } .
$$

We will hereafter refer to region $R$ as the one in the space $m_{W}-\xi \sigma_{\text {scalar }}^{\text {(nucleon) }}$ which is defined by Eqs. (4),(5). The region $R$ represents the sensitivity region already under exploration with present detectors.

Our analysis, based on an interpretation of experimental data in terms of relic neutralinos, will show by how much the WIMP direct searches probe the supersymmetric parameter space. We remark that, in the case of neutralinos, the assumption about the dominance of the coherent cross section over the spin-dependent one is, in general, largely satisfied, except for values of $\sigma_{\text {scalar }}^{\text {(nucleon) }}$ which are far below the present experimental reach [1]. 
The present analysis will be performed in the framework of various schemes, from those based on universal or nonuniversal supergravity, with SUSY parameters defined at the grand unification scale to an effective supersymmetric model defined at the electroweak (EW) scale. This is discussed in Sec. II, where we also specify the values employed here for the Higgs-quark-quark and the neutralino-quark-squark couplings. These quantities are subject to sizable uncertainties, as was stressed in Ref. [28], which triggered a reconsideration of this important point in a number of subsequent papers $[12,13,29]$.

The most important properties to be established for the relic neutralinos, which are entailed in the exploration by WIMP direct searches, concern their cosmological properties. Here, we perform a general analysis which is not limited to a restricted range of the cosmological matter abundance, $\Omega_{m} h^{2}\left(\Omega_{m}\right.$ is the matter cosmological density divided by the critical density and $h$ is the Hubble constant in units of $100 \mathrm{~km} \mathrm{~s}^{-1} \mathrm{Mpc}^{-1}$ ). Instead, we derive the average and local cosmological properties of the SUSY configurations from experimental determinations of $\sigma_{\text {scalar }}^{\text {(nucleon) }}$, without any a priori requested range on $\Omega_{m} h^{2}$. On the basis of the results of our evaluations in the various supersymmetry models, we discuss when the relic neutralino does or does not saturate the expected amount of the local and of the average amount of total dark matter. Our results and conclusions are presented in Secs. III and IV, respectively.

\section{SUPERSYMMETRIC MODELS}

The calculations presented in this paper are based on the minimal supersymmetric extension of the standard model (MSSM), in a variety of different schemes. The essential elements of the MSSM are described by a Yang-Mills Lagrangian, the superpotential, which contains all the Yukawa interactions between the standard and supersymmetric fields, and by the soft-breaking Lagrangian, which models the breaking of supersymmetry. To fix the notations, we write down explicitly the soft supersymmetry breaking terms

$$
\begin{aligned}
-\mathcal{L}_{\text {soft }}= & \sum_{i} m_{i}^{2}\left|\phi_{i}\right|^{2}+\left\{\left[A_{a b}^{l} h_{a b}^{l} \widetilde{L}_{a} H_{1} \widetilde{R}_{b}+A_{a b}^{d} h_{a b}^{d} \widetilde{Q}_{a} H_{1} \widetilde{D}_{b}\right.\right. \\
& \left.\left.+A_{a b}^{u} h_{a b}^{u} \widetilde{Q}_{a} H_{2} \widetilde{U}_{b}+\text { H.c. }\right]-B \mu H_{1} H_{2}+\text { H.c. }\right\} \\
& +\sum_{i} M_{i}\left(\lambda_{i} \lambda_{i}+\bar{\lambda}_{i} \bar{\lambda}_{i}\right)
\end{aligned}
$$

where the $\phi_{i}$ are the scalar fields, the $\lambda_{i}$ are the gaugino fields, $H_{1}$ and $H_{2}$ are the two Higgs fields, $\widetilde{Q}$ and $\widetilde{L}$ are the doublet squark and slepton fields, respectively, and $\widetilde{U}, \widetilde{D}$, and $\widetilde{R}$ denote the $\mathrm{SU}(2)$-singlet fields for the up-squarks, down-squarks and sleptons. In Eq. (6), $m_{i}$ and $M_{i}$ are the mass parameters of the scalar and gaugino fields, respectively, and $A$ and $B$ denote trilinear and bilinear supersymmetry breaking parameters, respectively. The Yukawa interactions are described by the parameters $h$, which are related to the masses of the standard fermions by the usual expressions, e.g., $m_{t}=h^{t} v_{2}, m_{b}=h^{b} v_{1}$, where $v_{i}=\left\langle H_{i}\right\rangle$.
Implementation of this model within a supergravity scheme leads naturally to a set of unification assumptions at a grand unified theory (GUT) scale $M_{\mathrm{GUT}}$, (i) unification of the gaugino masses $M_{i}\left(M_{\mathrm{GUT}}\right) \equiv m_{1 / 2}$, (ii) universality of the scalar masses with a common mass denoted by $m_{0}$, $m_{i}\left(M_{\mathrm{GUT}}\right) \equiv m_{0}$; (iii) universality of the trilinear scalar couplings, $A^{l}\left(M_{\mathrm{GUT}}\right)=A^{d}\left(M_{\mathrm{GUT}}\right)=A^{u}\left(M_{\mathrm{GUT}}\right) \equiv A_{0} m_{0}$.

This scheme will be denoted here as universal supergravity (SUGRA) (or simply SUGRA). The relevant parameters of the model at the electroweak (EW) scale are obtained from their corresponding values at the $M_{G U T}$ scale by running these down according to the renormalization group equations (RGE). By requiring that the electroweak symmetry breaking is induced radiatively by the soft supersymmetry breaking, one finally reduces the model parameters to five: $m_{1 / 2}, m_{0}, A_{0}, \tan \beta\left(\equiv v_{2} / v_{1}\right)$, and sign $\mu$. In the present paper, these parameters are varied in the following ranges: $50 \mathrm{GeV} \leqslant m_{1 / 2} \leqslant 1 \mathrm{TeV}, m_{0} \leqslant 1 \mathrm{TeV},-3 \leqslant A_{0} \leqslant$ $+3,1 \leqslant \tan \beta \leqslant 50$. Notice that a common upper extreme for the mass parameters has been used, and generically set at the value of $1 \mathrm{TeV}$, as a typical scale beyond which the main attractive features of supersymmetry fade away. However, fine-tuning arguments actually set different bounds for $m_{0}$ and $m_{1 / 2}$ [in universal SUGRA and in nonuniversal SUGRA (NUSUGRA)] [27]: $m_{1 / 2} \lesssim$ hundreds of GeV, whereas $m_{0}$ $\$ 2-3 \mathrm{TeV}$. In the present paper we did not look specifically into the $m_{0} \sim 2-3 \mathrm{TeV}$ window; in Ref. [30] phenomenology of relic neutralinos in this large $m_{0}$ regime has been analyzed [31].

Models with unification conditions at the GUT scale represent an appealing scenario; however, some of the assumptions listed above, particularly (ii) and (iii), are not very solid, since, as was already emphasized some time ago [32], universality might occur at a scale higher than $M_{G U T}$ $\sim 10^{16} \mathrm{GeV}$, e.g., at the Planck scale. More recently, the possibility that the initial scale for the RGE running, $M_{I}$, might be smaller than $M_{G U T} \sim 10^{16}$ has been raised [14,33], on the basis of a number of string models (see for instance the references quoted in [14]). In Ref. [14] it is stressed that $M_{I}$ might be anywhere between the EW scale and the Planck scale with significant consequences for the size of the neutralino-nucleon cross section.

An empirical way of taking into account the uncertainty in $M_{I}$ consists in allowing deviations in the unification conditions at $M_{G U T}$. For instance, deviations from universality in the scalar masses at $M_{G U T}$, which split $M_{H_{1}}$ from $M_{H_{2}}$ may be parametrized as

$$
M_{H_{i}}^{2}\left(M_{G U T}\right)=m_{0}^{2}\left(1+\delta_{i}\right)
$$

This is the case of nonuniversal SUGRA (NUSUGRA) that we considered in Refs. [7,27], and that we analyze again in this paper. Here the parameters $\delta_{i}$ which quantify the departure from universality for the $M_{H_{i}}^{2}$ will be varied in the range $(-2,+2)$. Deviations from universality in the Higgs masses have recently been considered also in Ref. [15]. Further extensions of deviations from universality in SUGRA 
models which include squark and/or gaugino masses are discussed, for instance, in [13,29].

The large uncertainties involved in the choice of the scale $M_{I}$ make the use of SUGRA schemes rather problematic and unpractical; the originally appealing feature of a universal SUGRA with few parameters fails because of the need to take into consideration the variability of $M_{I}$ or, alternatively, to add new parameters which quantify the various deviation effects from universality at the GUT scale. It appears more convenient to work with a phenomenological SUSY model whose parameters are defined directly at the electroweak scale. We denote here this effective scheme of MSSM by EMSSM. This provides, at the EW scale, a model defined in terms of a minimum number of parameters: only those necessary to shape the essentials of the theoretical structure of an MSSM, and of its particle content. Once all experimental and theoretical constraints are implemented in this EMSSM model, one may investigate its compatibility with SUGRA schemes at the desired $M_{I}$.

In the EMSSM scheme we consider here, we impose a set of assumptions at the electroweak scale: (a) all trilinear parameters are set to zero except those of the third family, which are unified to a common value $A$, (b) all squark softmass parameters are taken degenerate, $m_{\tilde{q}_{i}} \equiv m_{\tilde{q}}$, (c) all slepton soft-mass parameters are taken degenerate, $m_{\tilde{l}_{i}} \equiv m_{\tilde{l}}$, (d) the U(1) and $\mathrm{SU}(2)$ gaugino masses, $M_{1}$ and $M_{2}$, are assumed to be linked by the usual relation $M_{1}$ $=(5 / 3) \tan ^{2} \theta_{W} M_{2}$ (this is the only GUT-induced relation we are using, since gaugino mass unification appears to be better motivated than scalar masses universality). As a consequence, the supersymmetric parameter space consists of seven independent parameters. We choose them to be $M_{2}, \mu, \tan \beta, m_{A}, m_{\tilde{q}}, m_{\tilde{l}}, A$, and vary these parameters in the following ranges: $50 \mathrm{GeV} \leqslant M_{2} \leqslant 1 \mathrm{TeV}, \quad 50 \mathrm{GeV} \leqslant|\mu|$ $\leqslant 1 \mathrm{TeV}, \quad 80 \mathrm{GeV} \leqslant m_{A} \leqslant 1 \mathrm{TeV}, \quad 100 \mathrm{GeV} \leqslant m_{\tilde{q}}, m_{\tilde{l}}$ $\leqslant 1 \mathrm{TeV},-3 \leqslant A \leqslant+3,1 \leqslant \tan \beta \leqslant 50 \quad\left(m_{A}\right.$ is the mass of the $C P$-odd neutral Higgs boson).

The EMSSM scheme proves very manageable for the SUSY phenomenology at the EW scale; as such, it has been frequently used in the literature in connection with relic neutralinos (often with the further assumption of slepton-squark mass degeneracy: $m_{\tilde{q}}=m_{\tilde{l}}$ ) $[6,9,11,34-36]$. Notice that we are not assuming here slepton-squark mass degeneracy. In the scatter plots given in this paper only configurations with $m_{\tilde{q}} \geqslant m_{\tilde{l}}$ are shown. This mass hierarchy is reminiscent of what is usually obtained in SUGRA schemes, although in our EMSSM it is not necessarily so. It is worth reporting that some configurations with inverse hierarchy $m_{\tilde{q}} \leqslant m_{\tilde{l}}$ produce some increase in $\xi \sigma_{\text {scalar }}^{\text {(nuclen) }}$ at low $m_{\chi}$ values (see the discussion after Fig. 5 in Sec. III).

We recall that even much larger extensions of the supersymmetric models could be envisaged: for instance, nonunification of the gaugino masses [29,37], and schemes with $C P$-violating phases [38]. Here we limit our considerations to the schemes previously defined: universal SUGRA, NUSUGRA, EMSSM.

The neutralino is defined as the lowest-mass linear superposition of photino $(\tilde{\gamma})$, zino $(\widetilde{Z})$, and the two Higgsino states $\left(\widetilde{H}_{1}^{0}, \widetilde{H}_{2}^{0}\right), \chi \equiv a_{1} \tilde{\gamma}+a_{2} \widetilde{Z}+a_{3} \widetilde{H}_{1}^{0}+a_{4} \widetilde{H}_{2}^{0}$. Hereafter, the nature of the neutralino is classified in terms of a parameter $P$, defined as $P \equiv a_{1}^{2}+a_{2}^{2}$. The neutralino is called a gaugino when $P>0.9$, a Higgsino when $P<0.1$, mixed otherwise.

For more details concerning theoretical aspects involved in our calculations and the way in which the experimental constraints due to $b \rightarrow s+\gamma$ is implemented we refer to Refs. [7,9]. Accelerators data on supersymmetric and Higgs boson searches (CERN $e^{+} e^{-}$collider LEP2 and Collider Detector $\mathrm{CDF}$ at Fermilab) provide now rather stringent bounds on supersymmetric parameters. CDF bounds are taken from [39]. The new LEP2 bounds are taken from $[26,40]$; these constrain the configurations of relevance for relic neutralinos more severely as compared, for instance, with those considered in Ref. [9].

The results for the neutralino relic abundance have been obtained with the procedure indicated in Ref. [41]. The neutralino-nucleon cross section has been calculated with the formulas reported in Refs. [6,28]. As discussed in the Introduction, this cross section suffers from significant uncertainties in the size of Higgs-quark-quark and squark-quarkneutralino couplings. In this paper we use for these quantities what we have defined as set 1 and set 2 in Ref. [28] to which we refer for details. Here we only report the values of the quantities $m_{q}\langle\bar{q} q\rangle$ for the two sets:

$$
\begin{aligned}
\text { Set 1: } \quad m_{l}\langle\bar{l} l\rangle & =23 \mathrm{MeV}, \\
m_{s}\langle\bar{s} s\rangle & =215 \mathrm{MeV}, \\
m_{h}\langle\bar{h} h\rangle & =50 \mathrm{MeV} . \\
\text { Set } 2: \quad m_{l}\langle\bar{l} l\rangle & =30 \mathrm{MeV}, \\
m_{s}\langle\bar{s} s\rangle & =435 \mathrm{MeV}, \\
m_{h}\langle\bar{h} h\rangle & =33 \mathrm{MeV} .
\end{aligned}
$$

In Eqs. (8),(9) $l$ stands for light quarks, $s$ is the strange quark and $h=c, b, t$ denotes heavy quarks. For the light quarks, we have defined $m_{l}\langle\bar{l} l\rangle \equiv \frac{1}{2}\left[m_{u}\langle\bar{u} u\rangle+m_{d}\langle\bar{d} d\rangle\right]$. Set 1 and set 2 bracket, only partially, the present uncertainties. In Ref. [28] we also considered the consequences of using a more extreme set of values. It is worth recalling that the quantity $m_{s}\langle\bar{s} s\rangle$ is crucial in establishing the size of $\sigma_{\text {scalar }}^{\text {(nucleon) }}$ [42].

The results shown in the next section are obtained with the same numerical codes employed in our previous papers $[6-9,28]$, but take into account all new accelerator data.

\section{RESULTS}

We turn now to the presentation of our results. In Figs. $1 \mathrm{a}-1 \mathrm{c}$ we give the scatter plots for $\sigma_{\text {scalar }}^{\text {(nuclen) }}$ versus $\Omega_{\chi} h^{2}$ for the three different schemes: universal SUGRA, nonuniversal SUGRA and EMSSM. For the SUGRA schemes we only display the results corresponding to positive values of $\mu$, 
SUGRA $\mu>0 \quad($ set 1$)$

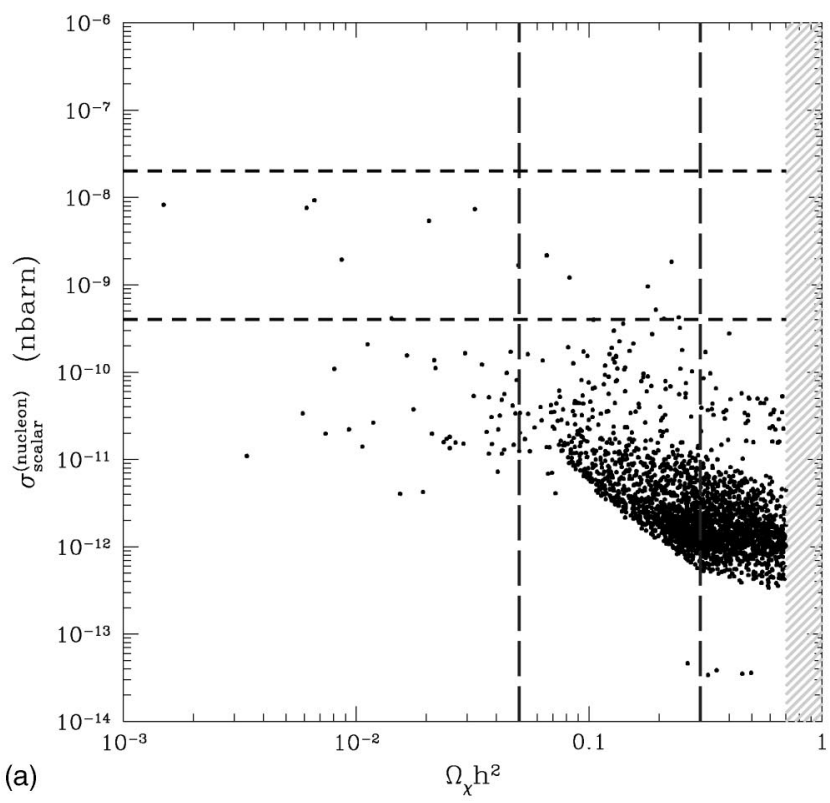

NUSUGRA $\mu>0($ set 1$)$

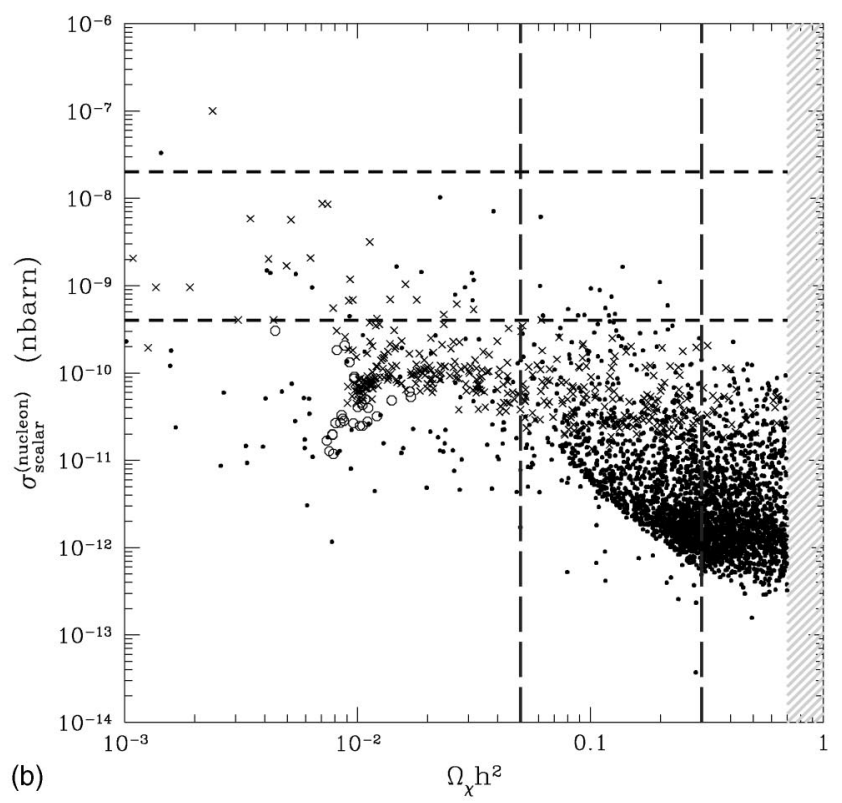

$\operatorname{EMSSM}(\operatorname{set} 1)$

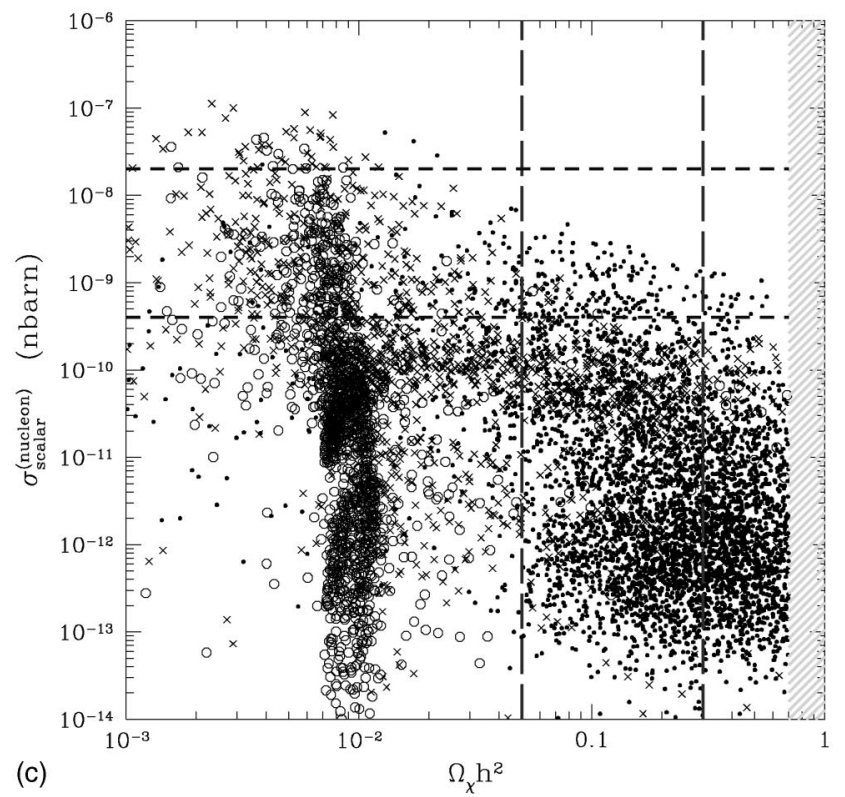

FIG. 1. (a) Scatter plot of $\sigma_{\text {scalar }}^{\text {(nucleon) }}$ versus $\Omega_{\chi} h^{2}$ for universal SUGRA. Set 1 for the quantities $m_{q}\langle\bar{q} q\rangle$ 's is employed. Only configurations with positive $\mu$ are shown and $m_{\chi}$ is taken in the range of Eq. (4). The two horizontal lines bracket the sensitivity region defined by Eq. (5). The two vertical lines denote the range $0.05 \leqslant \Omega_{m} h^{2} \leqslant 0.3$. The region above $\Omega_{\chi} h^{2}=0.7$ is excluded by current limits on the age of the universe. All points of this scatter plot denote gaugino configurations. (b) Scatter plot of $\sigma_{\text {scalar }}^{\text {(nucleon }}$ versus $\Omega_{\chi} h^{2}$ for NUSUGRA. Notations as in (a), except that here the scatter plot contains neutralinos of various configurations: dots denote gauginos, circles denote higgsinos and crosses denote mixed configurations. (c) Scatter plot of $\sigma_{\text {scalar }}^{\text {(nucleon) }}$ versus $\Omega_{\chi} h^{2}$ for EMSSM. Notations as in (b). Both signs of $\mu$ are shown.

since, for negative values, the constraint on $b \rightarrow s+\gamma$ implies a large suppression of $\sigma_{\text {scalar }}^{\text {(nucleon) }}$. The two horizontal lines bracket the sensitivity region defined by Eq. (5). The two vertical lines denote a favorite range for $\Omega_{m} h^{2}, 0.05$ $\leqslant \Omega_{m} h^{2} \leqslant 0.3$, as derived from a host of observational data. According to the most recent determinations [43], the lower bound on $\Omega_{m} h^{2}$ is approaching the value 0.08 . However, due to the still unsettled situation as regards determinations of the matter density in the universe and of the Hubble constant, a conservative attitude seems advisable. Anyway, we stress that in the present paper we are not restricting ourselves to any particular interval of $\Omega_{m} h^{2}$. Only some features of Fig. 5 
$\operatorname{EMSSM}(\operatorname{set} 2)$

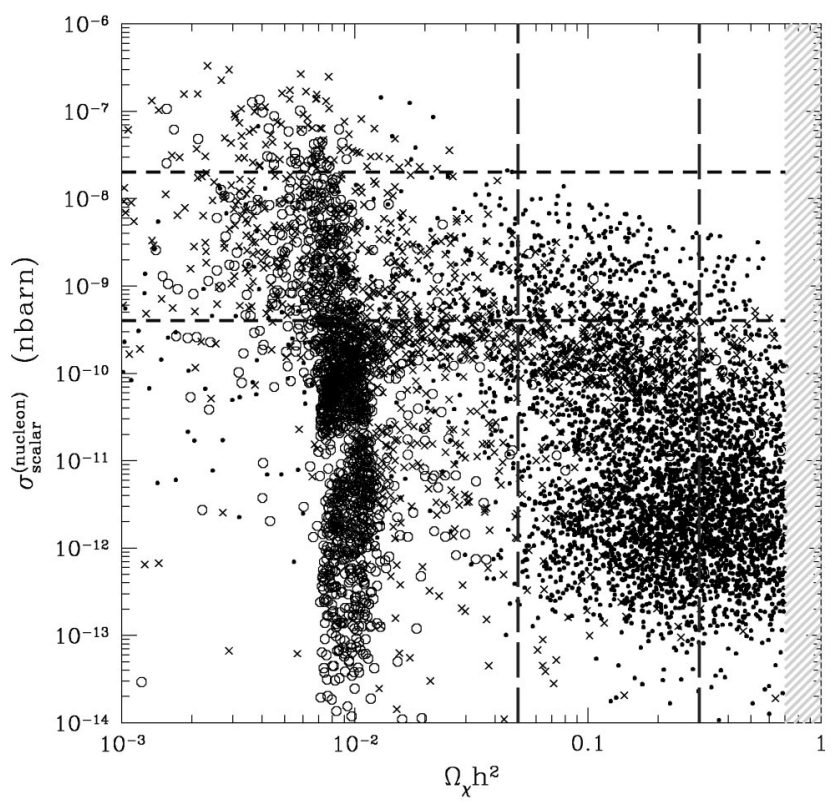

FIG. 2. Same as in Fig. 1(c), except that here set 2 for the quantities $m_{q}\langle\bar{q} q\rangle$ 's is employed instead of set 1 .

depend on the actual value employed for the minimum amount of matter necessary to reproduce the halo properties correctly.

Figures $1 \mathrm{a}-1 \mathrm{c}$ provide a first relevant result of our analysis: the present experimental sensitivity in WIMP direct searches allows the exploration of supersymmetric configurations of cosmological interest, also in the constrained SUGRA scheme. It is remarkable that the upper frontier of the scatter plots is not significantly different in the three different models, although the region of experimental sensitivity and cosmological interest is covered with an increasingly larger variety of supersymmetric configurations as one moves from SUGRA to NUSUGRA and to EMSSM. This latter fact is expected from the intrinsic features of the various schemes. This point will be further discussed later on, in connection with Fig. 4. Figure 2 shows what is the effect of using set 2 instead of set 1 for the quantities $m_{q}\langle\bar{q} q\rangle$ 's in EMSSM.

Once a measurement of the quantity $\rho_{\chi} \cdot \sigma_{\text {scalar }}^{\text {(nucleon) }}$ is performed, values for the local density $\rho_{\chi}$ versus the relic abundance $\Omega_{\chi} h^{2}$ may be deduced by proceeding in the following way [28]: (1) $\rho_{\chi}$ is evaluated as $\left[\rho_{\chi} \cdot \sigma_{\text {scalar }}^{\text {(nucleon) }}\right]_{\text {expt }} / \sigma_{\text {scalar }}^{\text {(nucleon) }}$, where $\left[\rho_{\chi} \cdot \sigma_{\text {scalar }}^{\text {(nuclen })}\right]_{\text {expt }}$ denotes the experimental value, and $\sigma_{\text {scalar }}^{\text {(nuclen }}$ is calculated as indicated above, (2) to each value of $\rho_{\chi}$ one associates the corresponding calculated value of $\Omega_{\chi} h^{2}$. The scatter plot in Fig. 3 is derived from the lowest value of the annualmodulation region of Ref. [3], $\left[\rho_{\chi} /\left(0.3 \mathrm{GeV} \mathrm{cm}^{-3}\right)\right.$ $\left.\times \sigma_{\text {scalar }}^{\text {(nucleon })}\right]_{\text {expt }}=1 \times 10^{-9}$ nbarn, and by taking $m_{\chi}$ in the range of Eq. (4). This plot, obtained in case of EMSSM, shows that the most interesting region, i.e., the one with $0.2 \mathrm{GeV} \mathrm{cm}^{-3} \leqslant \rho_{\chi} \leqslant 0.7 \mathrm{GeV} \mathrm{cm}^{-3}$ and $0.05 \leqslant \Omega_{m} h^{2}$
$\operatorname{EMSSM}(\operatorname{set} 1)$

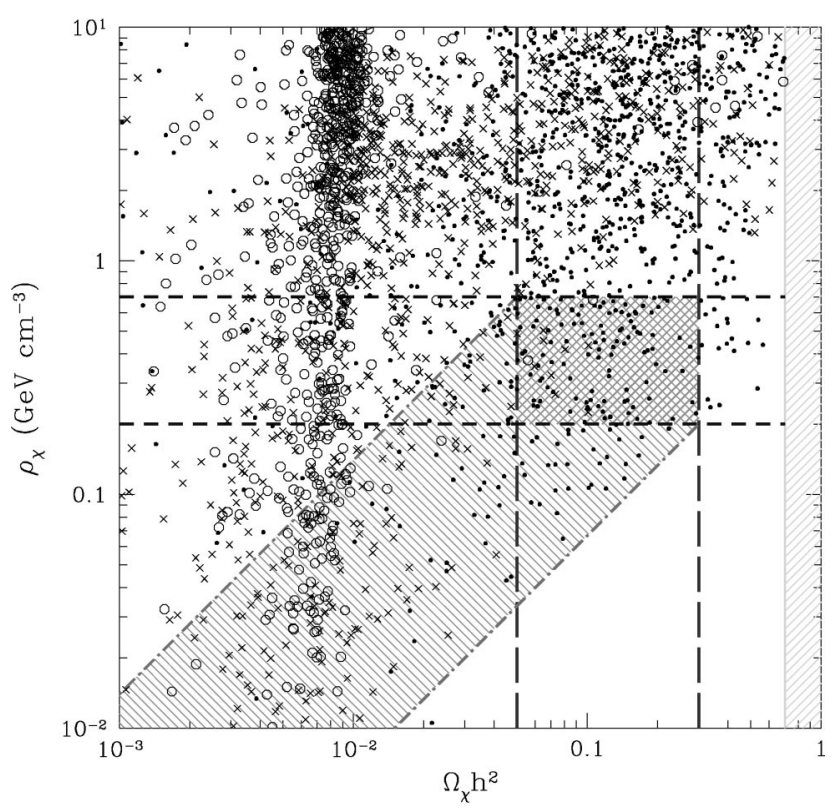

FIG. 3. Scatter plot of $\rho_{\chi}$ versus $\Omega_{\chi} h^{2}$. This plot is derived from the experimental value $\left[\rho_{\chi} /\left(0.3 \mathrm{GeV} \mathrm{cm}^{-3}\right) \cdot \sigma_{\text {scalar }}^{\text {(nucleon })}\right]_{\text {expt }}$ $=1 \times 10^{-9}$ nbarn and by taking $m_{\chi}$ in the range of Eq. (4), according to the procedure outlined in the text, in case of EMSSM. Set 1 for the quantities $m_{q}\langle\bar{q} q\rangle$ 's is employed. The two horizontal lines delimit the range $0.2 \mathrm{GeV} \mathrm{cm}^{-3} \leqslant \rho_{\chi} \leqslant 0.7 \mathrm{GeV} \mathrm{cm}^{-3}$; the two vertical ones delimit the range $0.05 \leqslant \Omega_{m} h^{2} \leqslant 0.3$. The region above $\Omega_{\chi} h^{2}=0.7$ is excluded by current limits on the age of the universe. The band delimited by the two slanted dot-dashed lines and simply hatched is the region where rescaling of $\rho_{l}$ applies. Dots denote gauginos, circles denote higgsinos, and crosses denote mixed configurations.

$\leqslant 0.3$ (cross-hatched region in the figure), is covered by SUSY configurations probed by the WIMP direct detection.

Let us examine the various sectors of Fig. 3. Configurations above the upper horizontal line are incompatible with the upper limit on the local density of dark matter in our Galaxy and must be disregarded. Configurations above the upper slanted dot-dashed line and below the upper horizontal solid line would imply a stronger clustering of neutralinos in our halo as compared to their average distribution in the Universe. This situation may be considered unlikely, since in this case neutralinos could fulfill the experimental range for $\rho_{\chi}$, but they would contribute only a small fraction to the cosmological cold dark matter content. For configurations which fall inside the band delimited by the slanted dotdashed lines and simply hatched in the figure, the neutralino would provide only a fraction of the cold dark matter at the level of local density and of the average relic abundance, a situation which would be possible, for instance, if the neutralino is not the unique cold dark matter particle component. To neutralinos belonging to these configurations one should assign a rescaled local density $\rho_{\chi}=\rho_{l} \times \Omega_{\chi} h^{2} /\left(\Omega_{m} h^{2}\right)_{\text {min }}$, where $\left(\Omega_{m} h^{2}\right)_{\min }$ is the minimum value of $\Omega_{m} h^{2}$ compatible with halo properties.

It is interesting to analyze the properties pertaining to the 
supersymmetric configurations which stay inside the favored region $0.2 \mathrm{GeV} \mathrm{cm}{ }^{-3} \leqslant \rho_{\chi} \leqslant 0.7 \mathrm{GeV} \mathrm{cm}^{-3}$ and 0.05 $\leqslant \Omega_{m} h^{2} \leqslant 0.3$ and to those which stay inside the corridor where rescaling applies (i.e. the corridor between the two slanted dot-dashed lines). In Figs. $4 \mathrm{a}-4 \mathrm{c}$ we give the scatter plots for all these configurations in the plane $m_{h}$ $-\tan \beta$ ( $m_{h}$ being the mass of the lightest $C P$-even neutral Higgs boson).

Let us make a few comments about these results. First, we note that a feature that was already pointed out in Ref. [7] is recovered: in SUGRA (Fig. 4a) only high values of $\tan \beta$, $\tan \beta \geq 40$, are involved in present direct detection experiments. Similar conclusions are also reached in more recent papers $[13,44]$. The occurrence of the lower bound $\tan \beta$ $\gtrsim 40$ in the SUGRA scheme is a consequence of RGE evolutions of the parameters and the nature of radiative electroweak symmetry breaking, which induce strong correlations among the parameters at the low energy scale [27]. In this class of models, couplings of the light Higgs boson $h$ to the $s$ quark can be enhanced only for large values of $\tan \beta$. As a consequence, the neutralino-nucleon cross section can be substantially large only when $\tan \beta \gtrsim 40$. Configurations displayed in Fig. 4a entail relatively light pseudoscalar Higgs $A, m_{A} \lesssim 200 \mathrm{GeV}, m_{0} \geqslant 350 \mathrm{GeV}$, and lightest stop and sbottom masses larger than about $460 \mathrm{GeV}$.

These features are somewhat (even though not completely) relaxed in the NUSUGRA scheme, where the nonuniversality in the Higgs sector allows for milder correlations among the parameters and among the low energy variables. This is especially true for the lower bound on $\tan \beta$, which now moves down to about 7 , as displayed in Fig. 4b. Also for the other parameters we have weaker bounds with respect to the SUGRA case. For instance, $m_{A}$ $\lesssim 450 \mathrm{GeV}, m_{0} \gtrsim 200 \mathrm{GeV}$, and lightest stop and sbottom masses larger than about $150 \mathrm{GeV}$ and $400 \mathrm{GeV}$, respectively.

In the EMSSM scheme most of the internal correlations of the model, which are typical of the supergravity inspired schemes, are not present. For instance, $\tan \beta$ and $m_{A}$ are now independent parameters. Also in this case, the most relevant information about configurations which give $\rho_{\chi}$ and $\Omega_{m} h^{2}$ inside the favored region defined above is provided by the $\tan \beta-m_{h}$ correlation, which is shown in Fig. $4 \mathrm{c}$. We notice that in EMSSM the lower bound on $\tan \beta$ is around 5. In Fig. $4 \mathrm{c}$ we also display, by a dashed line, what would be the boundary of the scatter plot, in case set 2 for the quantities $m_{q}\langle\bar{q} q\rangle$ 's is employed instead of set 1 . As for other relevant correlations, we find, for instance $m_{A} \lesssim 500 \mathrm{GeV}$ and/or $m_{\tilde{q}}$ $\$ 300 \mathrm{GeV}$.

As a final comment, we point out that in our calculations we have taken into account the experimental constraint on $\sin ^{2}(\alpha-\beta)[40]$. This limit is stronger than the one displayed in terms of $\tan \beta$ versus $m_{h}$ in Figs. $4 \mathrm{a}-4 \mathrm{c}$ and has the effect of depopulating the scatter plots without modifying their boundaries.

We wish to point out that, should the continuation of the LEP running provide some support in favor of a Higgs boson at a mass of about $115 \mathrm{GeV}$ [45], this would fit remarkably well within the configurations displayed in Fig. 4 [46]. It would be a case where accelerator measurements and WIMP searches would complement each other in providing a possible, consistent picture of physics beyond the standard model.

For sake of comparison with specific experimental results, we provide in Figs. $5 \mathrm{a}-5 \mathrm{c}$ the scatter plots for the quantity $\xi \sigma_{\text {scalar }}^{\text {(nucleon) }}$ versus $m_{\chi}$ in the various supersymmetric schemes. $\xi$ is taken to be $\xi=\min \left\{1, \Omega_{\chi} h^{2} / \Omega_{m} h^{2}\right\}$, in order to have rescaling in the neutralino local density, when $\Omega \chi h^{2}$ turns out to be less than $\left(\Omega_{m} h^{2}\right)_{\min }$ [here $\left(\Omega_{m} h^{2}\right)_{\min }$ is set to the value 0.05]. In universal SUGRA our results reach a maximum for $\xi \sigma_{\text {scalar }}^{\text {(nucleon })}$ at the level of about $10^{-9} \mathrm{nb}$, a feature which is in common, for instance, with the results of Refs. $[13,29,36]$. Lower values for the WIMP-nucleon cross section are found in evaluations where various inputs, each one having the effect of suppressing the value of $\xi \sigma_{\text {scalar }}^{\text {(nuclen) }}$, are employed concomitantly $[12,15]$, (i) low values for $\tan \beta, \tan \beta \leqslant 10$, (ii) small values for the quantity $m_{s}\langle\bar{s} s\rangle$, (iii) a tight lower bound on the neutralino relic abundance $\Omega_{\chi} h^{2}>0.1$. In fact, should we use the same inputs as in [12], we would obtain the scatter plot which stays below the dashed line displayed in Fig. 5a, in agreement with the results of Ref. [12].

However, we point out that, in general, in universal SUGRA, evaluations by various authors differ in some features, for instance in the position of the maximum of $\xi \sigma_{\text {scalar }}^{\text {(nuclen) }}$ in terms of $m_{\chi}$. This is likely to be due to the fact that this strict scheme is very sensitive to the specific ways in which various constraints (for instance, $\mathrm{b} \rightarrow \mathrm{s}+\gamma$ ) are implemented in the calculations.

In Figs. $5 \mathrm{a}-5 \mathrm{c}$ the solid line denotes the frontier of the $3 \sigma$ annual-modulation region of Ref. [3], when only the uncertainties in $\rho_{l}$ and in the dispersion velocity of a MaxwellBoltzmann distribution, but not the ones in other astrophysical quantities, are taken into account. As discussed in the Introduction, effects due to a possible bulk rotation of the dark halo or to an asymmetry in the WIMP velocity distribution would move this boundary towards higher values of $m_{\chi}$. Our results in Figs. 5a-5c show that the SUSY scatter plots reach up the annual-modulation region of Ref. [3], even with the current stringent bounds from accelerators (obviously, more easily in EMSSM than in SUGRA and NUSUGRA schemes).

In connection with the results shown in Fig. $5 \mathrm{c}$ for EMSSM, we further remark that, if configurations with the hierarchy $m_{\tilde{q}}<m_{\tilde{l}}$ are included, the scatter plot would rise by a factor of a few at $m_{\chi} \sim 50-90 \mathrm{GeV}$.

Finally, we recall that use of set 2 for the quantities $m_{q}\langle\bar{q} q\rangle$ 's instead of set 1 would entail an increase of about a factor 3 in all the scatter plots of Fig. 5.

\section{CONCLUSIONS}

In this work we have shown that the current direct experiments for WIMPs, when interpreted in terms of relic neutralinos, are indeed probing regions of the supersymmetric parameter space compatible with all present bounds from 
SUGRA $\mu>0($ set 1$)$

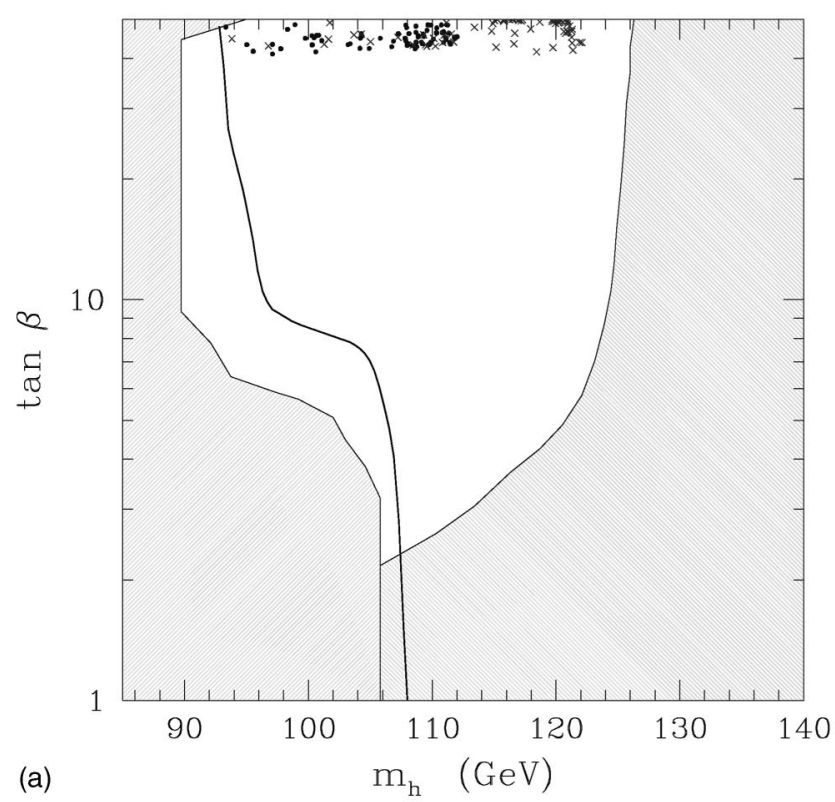

NUSUGRA $\mu>0($ set 1$)$

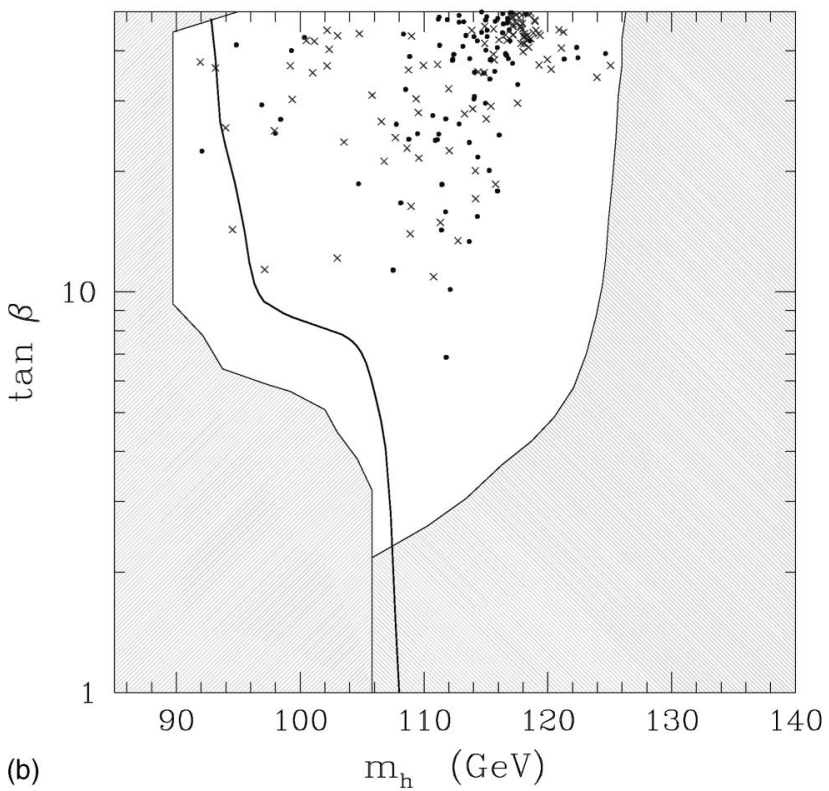

$\operatorname{EMSSM}(\operatorname{set} 1)$

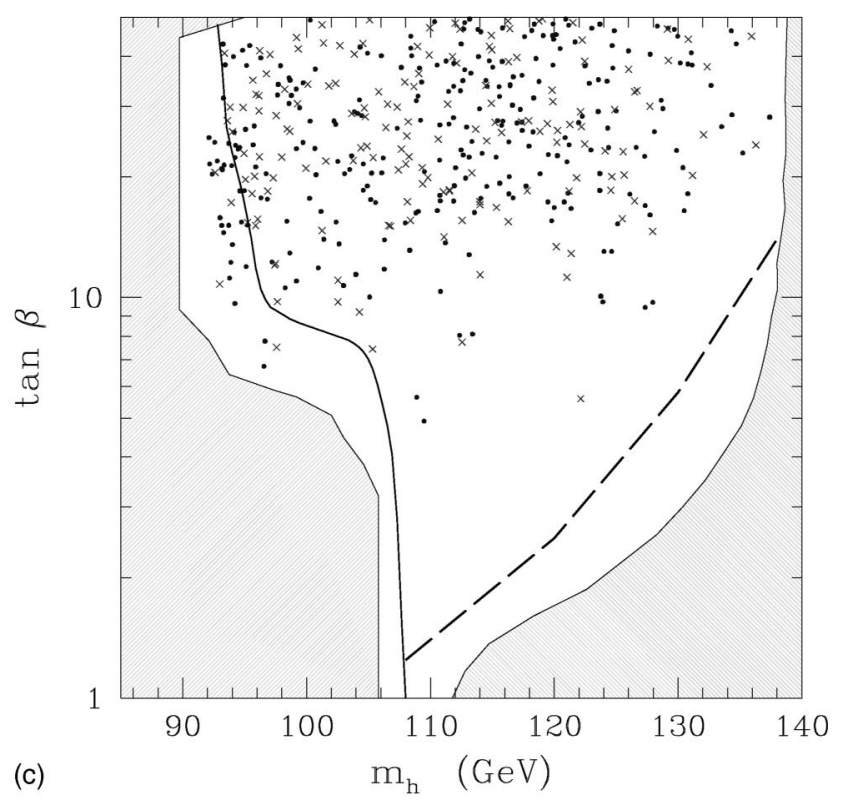

FIG. 4. (a) Scatter plot in the plane $m_{h}-\tan \beta$ of the SUGRA supersymmetric configurations which stay either inside the region $0.2 \mathrm{GeV} \mathrm{cm} \mathrm{cm}^{-3} \leqslant \rho_{\chi} \leqslant 0.7 \mathrm{GeV} \mathrm{cm} \mathrm{cm}^{-3}$ and $0.05 \leqslant \Omega_{m} h^{2} \leqslant 0.3$ or within the corridor of rescaling in the plane $\rho_{\chi}$ versus $\Omega_{\chi} h^{2}$. Set 1 for the quantities $m_{q}\langle\bar{q} q\rangle$ 's is employed. Crosses (dots) denote configurations with $\Omega_{\chi} h^{2}>0.05\left(\Omega_{\chi} h^{2}<0.05\right)$. The hatched region on the right is excluded by theory. The hatched region on the left is excluded by present data from LEP [26] and CDF [39]. The solid line represents the 95\% C.L. bound reachable at LEP2, in case of nondiscovery of a neutral Higgs boson. (b) Same as in (a) for configurations in NUSUGRA. (c) Same as in (a) for configurations in EMSSM. The dashed line denotes to which extent the scatter plot expands if set 2 for the quantities $m_{q}\langle\bar{q} q\rangle$ 's is used.

accelerators. We have quantified the extent of the exploration attainable by WIMP direct experiments in terms of various supersymmetric schemes, from a SUGRA scheme with unification assumptions at the grand unification scale to an effective model, EMSSM, at the electroweak scale. It has been stressed that, due to the large uncertainties in the unification assumptions in SUGRA schemes, the EMSSM framework turns out to be the most convenient model for neutralino phenomenology.

We have proved that part of the configurations probed by 
SUGRA $\mu>0(\operatorname{set} 1)$

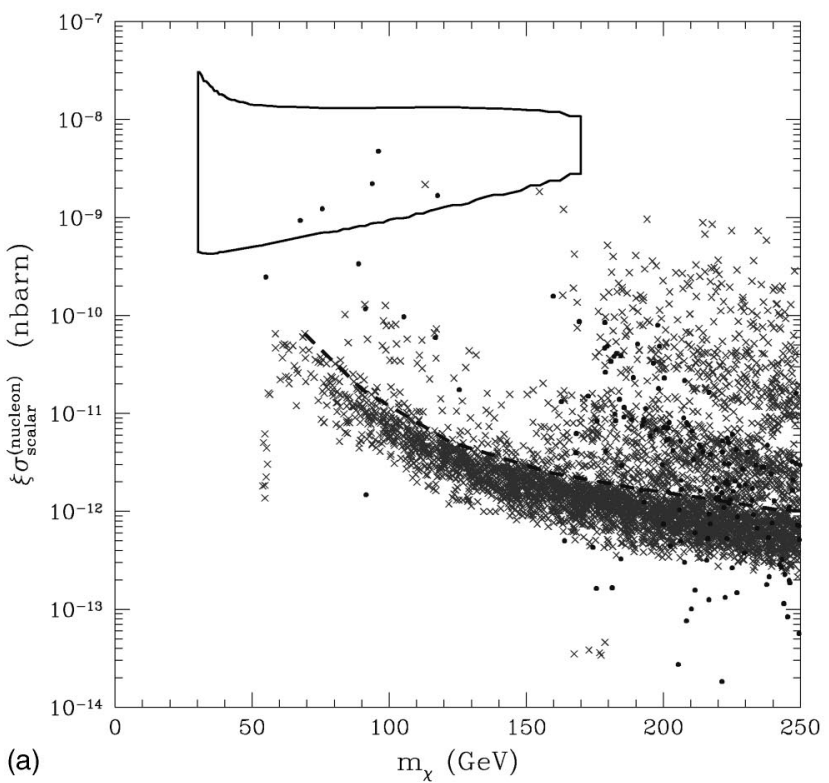

NUSUGRA $\mu>0(\operatorname{set} 1)$

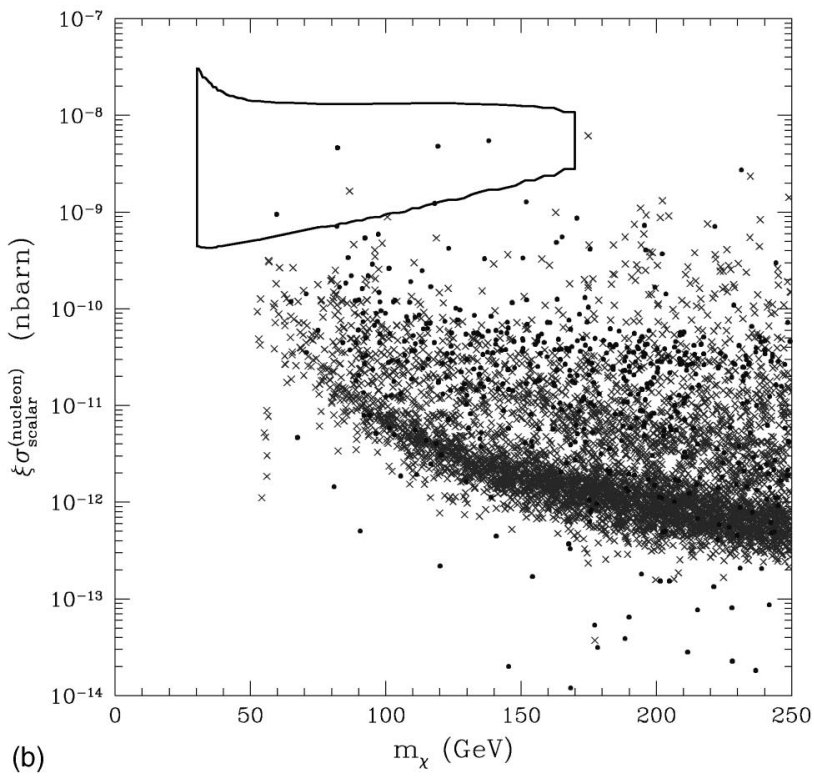

$\operatorname{EMSSM}(\operatorname{set} 1)$

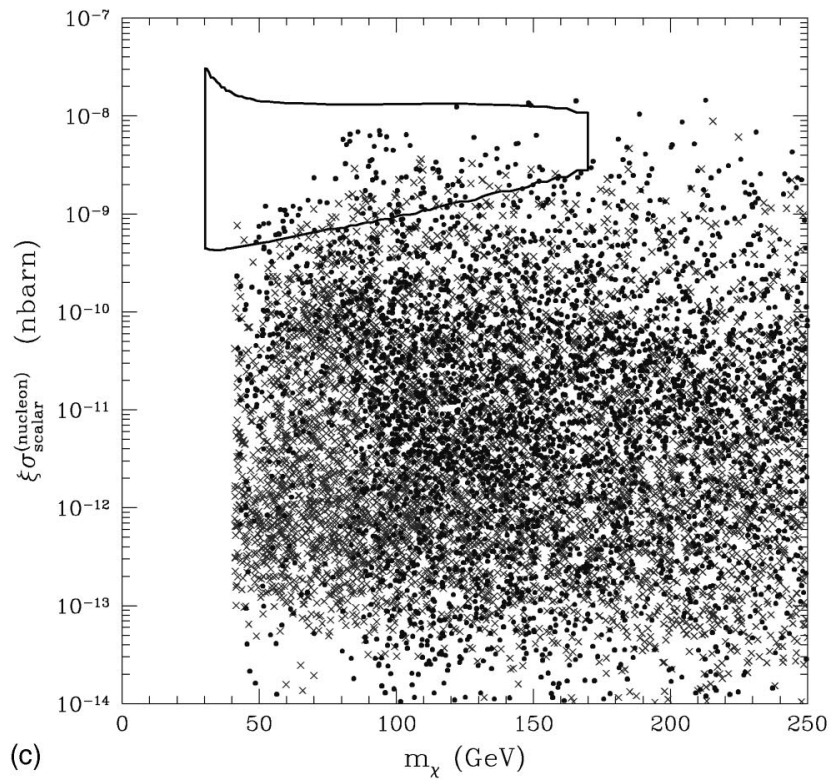

FIG. 5. (a) Scatter plot of $\xi \sigma_{\text {scalar }}^{\text {(nucleon) }}$ versus $m_{\chi}$ in case of universal SUGRA. Set 1 for the quantities $m_{q}\langle\bar{q} q\rangle$ 's is employed. Crosses (dots) denote configurations with $\Omega_{\chi} h^{2}>0.05\left(\Omega_{\chi} h^{2}<0.05\right)$. The dashed line delimits the upper frontier of the scatter plot, when the inputs of Ref. [12] are used. The solid contour denotes the $3 \sigma$ annual-modulation region of Ref. [3] (with the specifications given in the text). (b) Same as in (a) in case of NUSUGRA. (c) Same as in (a) in case of EMSSM.

WIMP experiments, and not disallowed by present accelerator bounds, entail relic neutralinos of cosmological interest. As discussed in the previous section, this result is at variance with the conclusions of some analyses recently appearing in the literature. Also neutralinos which might contribute only partially to the required amount of dark matter in the universe have been included in our analysis. The cosmological properties have been displayed in terms of a plot of the local density versus the average relic abundance, i.e., in a repre- sentation which proves particularly useful to summarize the properties of relic neutralinos.

We have noticed that a Higgs with a mass of about 115 $\mathrm{GeV}$, such as the one now under experimental investigation at LEP2, would fit remarkably well in the above scenario.

In our evaluations we have taken into account that the determination of the actual sensitivity region in terms of the WIMP-nucleon cross section and of the WIMP mass from the experimental data depends quite sizably on uncertainties 
of various origins, mainly, (i) possible effects due to a halo bulk rotation and/or to asymmetries in the WIMP velocities distribution, (ii) significant uncertainties in the determination of Higgs-quark-quark and neutralino-quark-squark couplings. We have stressed that all these effects have to be taken properly into account, when conclusions about comparison of theory with experiments are drawn.

\section{ACKNOWLEDGMENTS}

This work was partially supported by the Research Grants of the Italian Ministero dell'Università e della Ricerca Scientifica e Tecnologica (MURST) within the Astroparticle Physics Project. N.F. wishes to thank the kind hospitality of LAPTH in Annecy where part of this work has been done.
[1] A. Bottino, F. Donato, G. Mignola, S. Scopel, P. Belli, and A. Incicchitti, Phys. Lett. B 402, 113 (1997).

[2] For a review on WIMP direct searches see, for instance, A. Morales, Nucl. Phys. B (Proc. Suppl.) 87, 477 (2000).

[3] DAMA Collaboration, R. Bernabei et al., Phys. Lett. B 480, 23 (2000); ROM2F/2000-26, INFN/AE-00/10, http:// mercury.lngs.infn.it/lngs/preprint

[4] CDMS Collaboration, R. Abusaidi et al., Phys. Rev. Lett. 84, 5699 (2000).

[5] B. Bernabei et al., in the Proceedings of the PIC20 Conference, ROM2/2000-32, http://www.lngs.infn.it/lngs/htexts/ dama/dama7.html

[6] A. Bottino, F. Donato, N. Fornengo, and S. Scopel, Phys. Lett. B 423, 109 (1998); Phys. Rev. D 59, 095003 (1999).

[7] A. Bottino, F. Donato, N. Fornengo, and S. Scopel, Phys. Rev. D 59, 095004 (1999).

[8] P. Belli, R. Bernabei, A. Bottino, F. Donato, N. Fornengo, D. Prosperi, and S. Scopel, Phys. Rev. D 61, 023512 (2000).

[9] A. Bottino, F. Donato, N. Fornengo, and S. Scopel, Phys. Rev. D 62, 056006 (2000).

[10] R. Arnowitt and P. Nath, Phys. Rev. D 60, 044002 (1999).

[11] V.A. Bednyakov and H.V. Klapdor-Kleingrothaus, Phys. Rev. D 62, 043524 (2000).

[12] J. Ellis, A. Ferstl, and K.A. Olive, Phys. Lett. B 481, 304 (2000).

[13] E. Accomando, R. Arnowitt, B. Dutta, and Y. Santoso, Nucl. Phys. B585, 124 (2000).

[14] E. Gabrielli, S. Khalil, C. Muñz, and E. Torrente-Lujan, Phys. Rev. D 63, 025008 (2001).

[15] J. Ellis, A. Ferstl, and K.A. Olive, Phys. Rev. D 63, 065016 (2001).

[16] J. Binney and S. Tremaine, Galactic Dynamics (Princeton University Press, Princeton, 1987).

[17] E.I. Gates, G. Gyuk, and M.S. Turner, Astrophys. J. Lett. 449, L123 (1995).

[18] M.W. Goodman and E. Witten, Phys. Rev. D 31, 3059 (1986).

[19] R.H. Helm, Phys. Rev. 104, 1466 (1956).

[20] M. Kamionkowski and A. Kinkhabwala, Phys. Rev. D 57, 3256 (1998).

[21] F. Donato, N. Fornengo, and S. Scopel, Astropart. Phys. 9, 303 (1999).

[22] J.D. Vergados, Phys. Rev. Lett. 83, 3597 (1999); Phys. Rev. D 62, 023519 (2000); P. Ullio and M. Kamionkowski, J. High Energy Phys. 03, 049 (2001).

[23] N.W. Evans, C.M. Carollo, and P.T. de Zeeuw, astro-ph/0008156.

[24] A.M. Green, Phys. Rev. D 63, 043005 (2001).

[25] At variance with static models for the galactic halo, ongoing infall models have been considered. One example is provided by the caustic ring model suggested by P. Sikivie, I.I. Tkachev, and Y. Wang, Phys. Rev. Lett. 75, 2911 (1995). For implications of this model for WIMP detection see, for instance, J.D. Vergados, hep-ph/0010151, and references therein.

[26] I.M. Fisk and K. Nagai, talks at the XXXth International Conference on High Energy Physics, Osaka, 2000, http:// www.ichep2000.hep.sci.osaka-u.ac.jp

[27] V. Berezinsky, A. Bottino, J. Ellis, N. Fornengo, G. Mignola, and S. Scopel, Astropart. Phys. 5, 1 (1996).

[28] A. Bottino, F. Donato, N. Fornengo, and S. Scopel, Astropart. Phys. 13, 215 (2000).

[29] A. Corsetti and P. Nath, hep-ph/0003186.

[30] J.L. Feng, K.T. Matchev, and F. Wilczek, Phys. Lett. B 482, 388 (2000).

[31] We thank Dr. J.L. Feng for a comment on this point.

[32] N. Polonsky and A. Pomarol, Phys. Rev. Lett. 73, 2292 (1994); Phys. Rev. D 51, 6532 (1995); M. Olechowski and S. Pokorski, Phys. Lett. B 334, 201 (1995); D. Metalliotakis and H.P. Nilles, Nucl. Phys. B435, 115 (1995); A. Pomarol and S. Dimopoulos, ibid. B453, 83 (1995); H. Murayama, talk given at the 4th International Conference on Physics Beyond the Standard Model, Lake Tahoe, 1994, hep-ph/9503392; J.A. Casas, A. Lleyda, and C. Muñoz, Phys. Lett. B 389, 305 (1996).

[33] S.A. Abel, B.C. Allanach, F. Quevedo, L.E. Ibáñez, and M. Klein, J. High Energy Phys. 12, 026 (2000).

[34] A. Bottino, V. de Alfaro, N. Fornengo, G. Mignola, and S. Scopel, Astropart. Phys. 1, 61 (1992).

[35] L. Bergström and P. Gondolo, Astropart. Phys. 6, 263 (1996).

[36] V. Mandic, A. Pierce, P. Gondolo, and H. Murayama, hep-ph/0008022 v2.

[37] M. Drees and X. Tata, Phys. Rev. D 43, 2971 (1991); K. Griest and L. Roszkowski, ibid. 46, 3309 (1992); S. Mizuta, D. Ng, and M. Yamaguchi, Phys. Lett. B 300, 96 (1993).

[38] See, for instance, M. Brhlik and G.L. Kane, Phys. Lett. B 437, 331 (1998); S. Khalil and Q. Shafi, Nucl. Phys. B564, 19 (1999); T. Falk, A. Ferstl, and K.A. Olive, Astropart. Phys. 13, 301 (2000); P. Gondolo and K. Freese, hep-ph/9908390; S.Y. Choi, hep-ph/9908397.

[39] CDF Collaboration, J.A. Valls, FERMILAB-Conf-99/263-E CDF; http://fnalpubs.fnal.gov/archive/1999/conf/Conf-99-263E.html

[40] ALEPH Collaboration, P.J. Donan, 2000, http://alephwww.cern.ch/ALPUB/seminar/lepc_mar2000/ lepc2000.pdf

[41] A. Bottino, V. de Alfaro, N. Fornengo, G. Mignola, and M. Pignone, Astropart. Phys. 2, 67 (1994). 
[42] G.B. Gelmini, P. Gondolo, and E. Roulet, Nucl. Phys. B351, 623 (1991).

[43] See, for instance, W. Freedman, talk at COSMO2K, Korea, 2000 .

[44] A.B. Lahanas, D.V. Nanopoulos, and V.C. Spanos, hep-ph/0009065.

[45] ALEPH Collaboration, D. Schlatter, DELPHI Collaboration, T. Camporesi, L3 Collaboration, J.J. Blaising, OPAL Collabo- ration, C. Rembser, at the special seminar at CERN 2000 (see links to the LEP experiments at http://cern.web.cern.ch/CERN/ Experiments.html).

[46] A. Bottino, N. Fornengo, and S. Scopel, hep-ph/0012377. For theoretical considerations about a Higgs boson of this mass in universal SUGRA see also J. Ellis, G. Ganis, D.V. Nanopoulos, and K.A. Olive, Phys. Lett. B 502, 171 (2001). 